\section{RF Supplementary Heating for \\ Toroidal Reactors}

In a toroidal configuration of the Tokamak or Stellarator type, two fundamental objectives have to be reached, the confinement, and the heating of a dense and hot plasma during a time interval $\tau_{E}$, so as to satisfy the condition: $n \tau \supseteq 10^{14}$ required by the Lawson criterion to reach the $D, T$ thermonuclear region.

In Tokamaks and, in part, in Stellerators, the induced toroidal current not only provides for plasma confinement and equilibrium, but also supplies energy through ohmic heating directly to the electrons and indirectly to the ions by electron-ion collisions.

It is universally admitted that this energy source is effective in raising the electron and ion temperatures to a few keV. Nevertheless, this collisional energy transfer is insufficient to heat the plasma of a future toroidal reactor to ignition temperatures. Consequently, considerable attention has to be given to the question of supplementary heating schemes which can raise the temperature reached by ohmic heating to the ignition temperature. As a result, for some years now, supplementary heating has begun to occupy the centre of the fusion programme.

In big Tokamaks like JET, T.F.T.R., $T_{20}$ and JT 60 where significant thermonuclear energy production is expected, the additional power needed lies between 20 and $100 \mathrm{MW}$ depending on the value of $n \tau_{E}$ which in its turn depends on the loss mechanism that is considered to be the most important : Bohm, pseudo-classical, trapped electron or trapped ion instabilities. It is however clear that in a first phase, the injected power will be smaller, of the order of $10 \mathrm{MW}$ and with a long pulse $(\sim 10 s)$. Notice that this is already a big jump when compared with the powers we are injecting now which are of the order of a few hundred $\mathrm{kW}$ during a time 10 to $100 \mathrm{~ms}$.

Presently, the two main supplementary heating methods are neutral beam injection and radio frequency (RF) heating processes, based respectively on particle-particle collisions and wave-particle interactions. Of these, radio frequency wave-plasma interactions are very attractive, covering as they do a large variety of frequency depen- dent mechanisms for the absorption by a plasma of the energy carried by a wave.

As appears in Fig. 1, there exist various domains of interest, subdivided according to decreasing wave lengths into three classes: low, high, and very high frequencies.

From the coupling view-point, the low frequency group utilizes loops, which can be put outside the plasma vessel (Toroidal Drift Magnetic Pumping) or inside, (as for Transit Time Cyclotron Resonant Heating.

\section{T. Consoli, Grenoble}

(Centre d'Etudes Nucléaires)

Magnetic Pumping, Alfen Waves Resonant Heating and Ion Cyclotron Resonant Heating). In the second and third groups (the high and very high frequencies) the waves are launched by guides. We must remark that for high toroidal magnetic field systems $B \supseteq 5 T$ and for large Tokamaks, $2 r \geqslant 3 \mathrm{~m}$, I.C.R. waves can also be launched by ridge or loaded guides.

The possibility of using external coils in the case of very low frequency heating as in T.D.M.P., or of launching

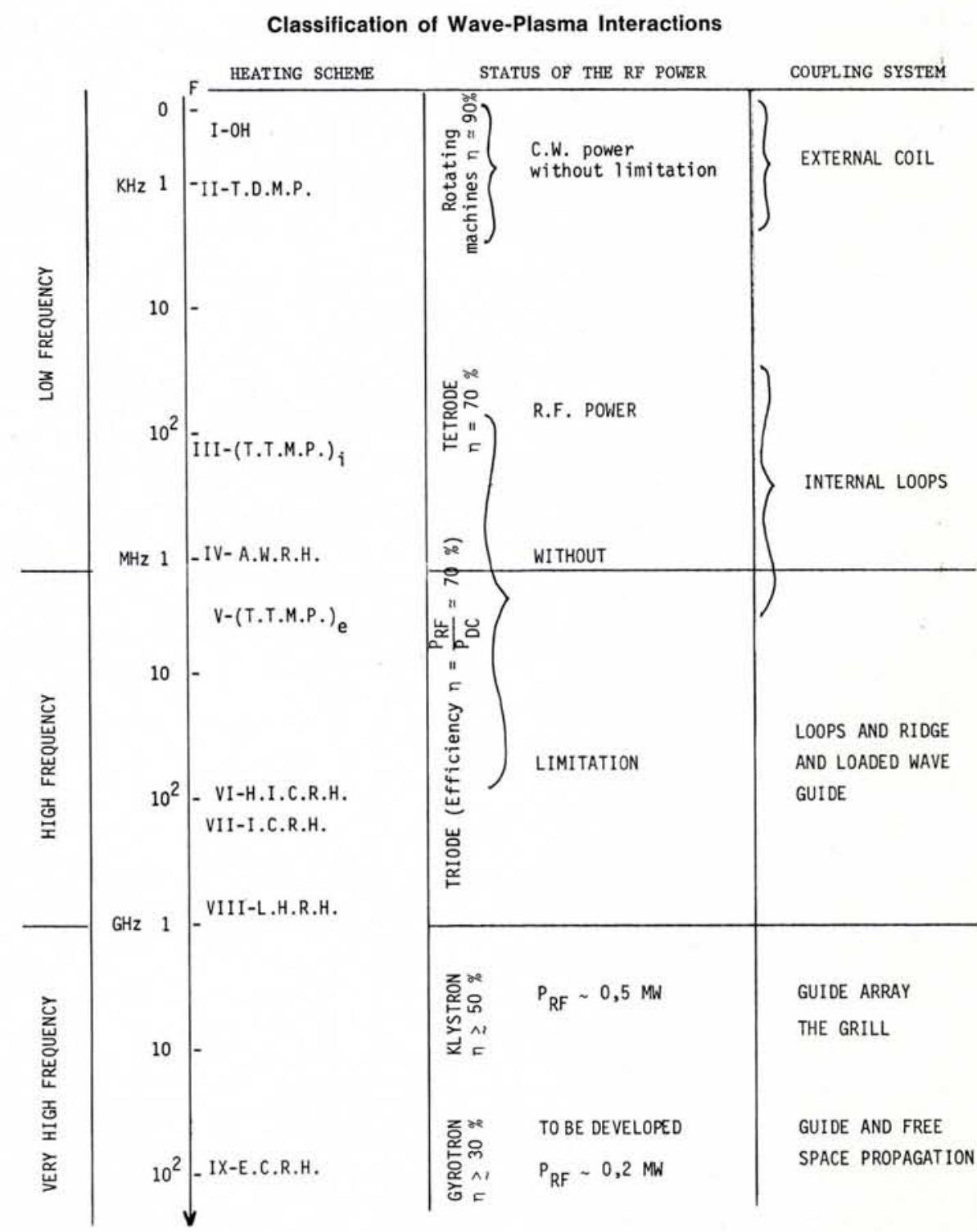

Fig. 1. Absorption mechanisms : I - Ohmic Heating, II - Toroidal Drift Magnetic Pumping, III - Transit Time Magnetic Pumping on the ions, IV - Alfven Waves Resonant Heating, V - Transit Time Magnetic Pumping on the electrons, VI - Hybrid lon Cyclotron Resonant Heating, VII - Magnetosonic Waves, VIII - Lower Hybrid Resonant Heating, IX - Electron 
the waves by guides directly connected to the toroidal chamber, as in the case of high frequency heating schemes (Lower Hybrid Resonant Heating) constitutes an undeniable advantage.

We intend to report mainly on three very promising methods: T.T.M.P., I.C.R.H. and L.H.R.H. which have been tested experimentally on toroidal devices. It seems, however, appropriate to mention first the potentialities of two other methods (T.D.M.P. and Electron Cyclotron Resonant Heating) which are, from a purely speculative viewpoint, very interesting for the future thermonuclear reactor.

\section{T.D.M.P. and E.C.R.H. \\ Heating Schemes}

These two methods, which are very attractive because of their launching systems, use respectively very low and very high frequencies and are at the state of proposals only. Indeed the first heating scheme has not been tested experimentally yet.

T.D.M.P. non-collisional RF heating was proposed recently by $E$. Canobbio (VIIIth I.A.E.A. Conf. Berchtesgaden, October 1976). It requires frequencies so low, (of the order of $\mathrm{kHz}$ ) that they can be supplied by a rotating machine, connected directly to coils (Fig. 2) external to the plasma vessel, which the waves penetrate easily. Suitably phased and modulated, they interact with the vertical component of the toroidal drift velocity.

The coupling coils may be the same coils used for the generation of the vertical magnetic field necessary for the plasma equilibrium. The electromagnetic energy carried by the excited waves is absorbed by Landau-like damping, if $\omega_{0}>v$, where $v$ is the collision frequency for the energy exchange of the resonant ions.

Fig. 2. The T.D.M.P. showing the position of the excitation coils.

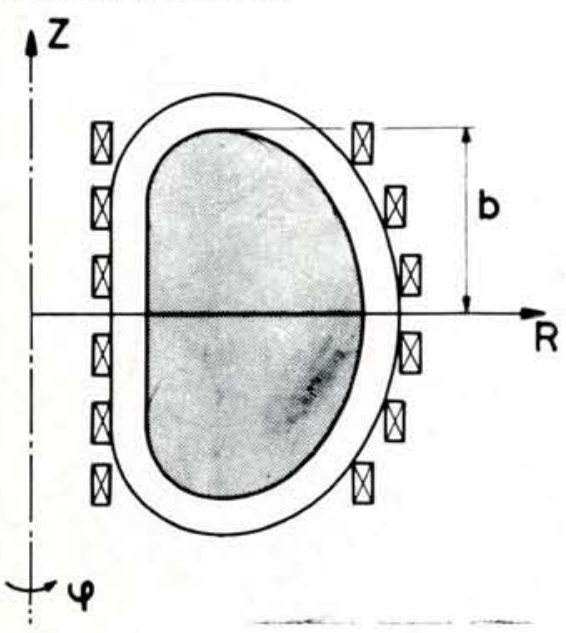

The space averaged power, absorbed by the ions of the plasma is proportional to $\left(n T_{i}\right)^{2}$ and to the square of the relative plasma displacement. This means that heating is more effective in dense and hot plasmas. The optimum frequency heating in the case of the JET parameters $\left(T_{i}=3 \mathrm{keV}\right.$, $R_{\mathrm{o}}=3 \mathrm{~m}, B=3$ Tesla, $b$, the vertical extension of the plasma $=1.2 \mathrm{~m}$, $b / a=1.6)$ is around $1 \mathrm{khz}$.

Cannobio also showed that by exploiting the existence within the plasma of the M.H.D. resonant surfaces, T.T.M.P. can be produced at similar low frequencies, but with much higher heating efficiency.

At the other end of the frequency spectrum E.C.R.H. has also very attractive features. This heating scheme requires the realization of a powerful millimetric source (the gyroklystron) now in development. Although preliminary experiments with $28 \mathrm{GHz}$ 200 kW CW will be undertaken in 1978 at Oak Ridge, a full scale realistic test at a frequency near to $10^{11} \mathrm{~Hz}(B$ (toroidal) $\sim 4 \mathrm{~T}, n_{e} \sim 10^{14} \mathrm{~cm}^{-3}$ ) will be possible only after 1980 .

The two physical mechanisms of wave energy absorption involved are well known. One is the collisionless damping of microwaves propagating at a frequency near the electron cyclotron frequency, and the other one is the linear conversion of electromagnetic wave energy into longitudinal waves near the upper hybrid resonance.

In an inhomogeneous plasma, the penetration of the wave to the resonant region through the external evanescent layer, requires the launching of the wave from the higher magnetic field side of the torus. The wave which has reached the resonance region, looses its energy by resonance or by Landau damping. In the resonance region, the electrons gain energy from the RF field through a stochastic process. The microwave power which is absorbed, is proportional to $n_{\mathrm{e}} f_{\mathrm{RF}} T_{\mathrm{e}}^{-1 / 2}$. The absorption length is very short, so that for example in a plasma of $T_{e}=10 \mathrm{keV}, n_{\mathrm{e}}=10^{14} \mathrm{~cm}^{-3}, t=$ $1.2 \times 10^{11}$, this distance is of the order of $\mathrm{mm}$, and consequently the microwave energy is absorbed in a single transit through the plasma.

This heating scheme has been tested with success at low magnetic field and low microwave power in mirror field configurations (CIRCE experiment at Grenoble and INTEREM at Oak-Ridge) and in Toroidal configurations (ELMO BUMBY TORUS at Oak-Ridge, $\mathrm{TM}_{3}$ Tokamak at Kurchatov).

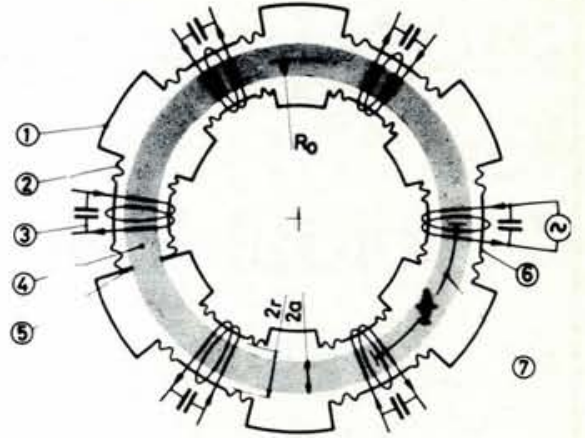

Fig. 3. The T.T.M.P. mounted round the liner of the Tokamak PETULA; 1 - observation port; 2 - stainless steel bellows; 3 - RF coils; 4 - the plasma ; 5 - the diaphragm ; 6 - an alumina sector ; 7 - halfwave length.

Experiments on large Tokamaks require powerful millimetric microwave generators, the development of which has been undertaken simultaneously in the U.S.S.R. (Prof. Gaponov Group at Gorki) and in the U.S. (the Varian Co. at Palo-Alto). When high power at higher frequencies becomes available after 1980, it will then be possible to shape the electron temperature, to control the genesis of the discharge in Tokamaks and to operate a Stellerator without ohmic current.

\section{Three Operational RF Heating Schemes}

While the two previously mentioned RF methods are still speculative, Transit Time Magnetic Pumping (T.T.M.P.), Ion Cyclotron Resonance Heating (I.C.R.H.) and Lower Hybrid Resonant Heating (L.H.R.H.) have reached, during these past few years, theoretically and experimentally a high degree of credibility.

\section{T.T.M.P.}

In this heating scheme, in the form tested up to now, the main toroidal magnetic field is modulated in space and time by currents circulating in antiphase in RF coils (Fig. 3) uniformly distributed along the torus.

The electromagnetic energy is transferred to the ions of the plasma if the thermal velocity of the ion is equal to the phase velocity of the wave. Here again the physical mechanism is of the Landau damping type. In large devices, the RF coils will have to be inside the metallic toroidal vessel. In spite of this disadvantage, this heating scheme is particularly interesting for large devices and for thermonuclear plasma. The heating is volumic and the electromagnetic energy is transferred first to the parallel component of the ion velocity, the electrons being heated by collision. Because of the uniform heating, 
the required density of energy is sufficiently low to avoid instabilities.

Collisions are needed for maintaining the Maxwellian shape of the velocity distribution function. The most interesting regime is the linear, for which the heating rate is proportional to $n_{\mathrm{i}} b^{2} T_{\mathrm{i}}^{3 / 2}$. It can be seen from this relationship, that the efficiency of this method, contrary to any other heating, including neutral beam injection, improves with the increasing thermal content of the plasma. If applied to a large device such as JET, a modulation ratio of the RF to the toroidal field of $2 \%$ and a power level of a few tens of MW will be sufficient to reach the ignition temperature.

The feasibility of this heating scheme (VIIIth European Conference, Prague 1977) has been demonstrated experimentally on the Tokamak PETULA (Grenoble Laboratory). The initial ion temperature of a plasma of mean density $2 \times 10^{13} \mathrm{~cm}^{-3}$ has been increased according to the theoretical law, without perturbing the plasma equilibrium, the energy distribution function, and the loop voltage. The ion temperature increase was measured by charge exchange and Doppler broadening of OVII line. No fast ions and impurities influx nor pumpout have been observed as in the previous experiments done in low density plasmas in Stellarator configuration.

The experimental results obtained allow us to consider this method as a cheap possible solution for large devices. Nevertheless, tests with internal coils on an intermediate size machine $\left(r=0.5 \mathrm{~m}, R=1.5 \mathrm{~m}, I_{\mathrm{T}}=\right.$ $0.5 \mathrm{MA}, B \sim 2 \mathrm{~T}, n_{\mathrm{e}}=5 \times 10^{13} \mathrm{~cm}^{-3}$ $\left.\left(T_{\mathrm{i}}\right)_{\mathrm{o}}=1 \mathrm{keV}\right)$ is needed to reach the required reliability for a machine of the reactor size. The internal coils will have to be protected with the same material as the first wall.

\section{I.C.R.H.}

The fast magnetosonic wave in deuterium and the ion hybrid resonance in both isotopes are two other promising approaches in the domain of I.C.R.H. In present devices in this frequency range, the coupling of the wave is facilitated by the existence of cavity eigenmode in the plasma loaded toroidal chamber, which allows efficient heating of the plasma. In large devices, the toroidal cavity becomes highly oversized so that the modes are so dense that their selection might be impossible. The coupling of RF energy to the fast toroidal eigenmodes is obtained generally by loops or by special wave guides.
The efficiency, the coupling, the mode tracking and the plasma heating have recently (1972-1977) been studied experimentally on the Tokamaks, ST and ATC at Princeton, $\mathrm{TM}_{1}$ and $\mathrm{T}_{4}$ at Kurchatov and the present situation can be summarized as follows :

- ST and ATC experiments have clearly demonstrated body heating.

- $\mathrm{TM}_{1}$ and $\mathrm{T}_{4}$ devices have shown the role, in the RF energy absorption, of a small percentage of hydrogen, when I.C.R. is excited. This effect will be exploited in the case of a two component $(D, T)$ plasma.

- The perpendicular energy distribution of the ions in all four experiments ST, ATC, TM1, $\mathrm{T}_{4}$ has an important tail.

For the energetic ions of this tail, strong neutral influx is observed, which serves to incease the rate of energy exchange between ions and electrons. Disruptive instabilities triggered in ST, ATC, $T_{4}$ are probably due to the impurity influx and not to the RF pulse. This influx can be attributed to wall bombardment, or to an uncontrolled displacement of the discharge, or to other, presently unknown, causes. The threshold for these instabilities was, $50 \mathrm{~kW}$ on $\mathrm{T}_{4}, 100 \mathrm{~kW}$ on ST, more than $140 \mathrm{~kW}$ on ATC.

In large devices, the density of toroidal eigenmodes in the frequency domain imposes mode selection and needs mode tracking. It seems thus impossible to extrapolate present experiments on fast wave mode physics to fusion devices without interme- diate steps. Experiments planned on large machines such as TFR 600, PLT, JET, will provide the appropriate conditions for the next steps.

\section{L.H.R.H.}

In the lower hybrid wave heating scheme, the waves are launched by wave guides, called the "grill", and propagate nearly perpendicular to the toroidal magnetic field in the plasma density gradient. In the region where the density corresponds to the lower hybrid frequency, the wave exhibits resonance and the fields become very large. The grill is a simple and elegant solution proposed first by $P$. Lallia and consists of an array of 2, 4 or more guides mounted with their small side parallel to the toroidal magnetic field. The phasing between the single elements of the grill is adjustable, so as to excite in the toroidal vessel a retarded wave. By means of suitable size and phase choice, the electrons or the ions can be heated.

An excellent experimental verification of Brambilla's grill theory has been obtained on the Princeton linear device $\mathrm{H}_{1}$ with 2- and 4-guide arrays and with a 2-guide grill on the PETULA Tokamak at the Grenoble Laboratory. The behaviour of the coupling system was, as predicted by the theory, determined by the plasma parameters near the antenna. A reflection coefficient of $99 \%$ has been obtained when the grill was well matched.

Ion heating has been observed on the Tokamak ATC (at Princeton), FTI

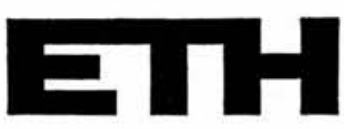

Applications are invited for occupancy of a chair in APPLIED PHYSICS

at the Physics Department

of the Swiss Federal Institute of Technology in Zurich.

Duties of the new professor will include teaching and research in applied physics, with emphasis on applied physics of metals.

The successful applicant will have several years of relevant experience and proven ability to perform and direct research. He is expected to teach at both undergraduate and graduate levels and to cooperate with his colleagues in the department.

Applications should be submitted with curriculum vitae and list of publications before March 31, 1978 to the President of the Swiss Federal Institute of Technology Zurich, Prof. Dr. H. Ursprung, 8092 Zurich, Switzerland. 


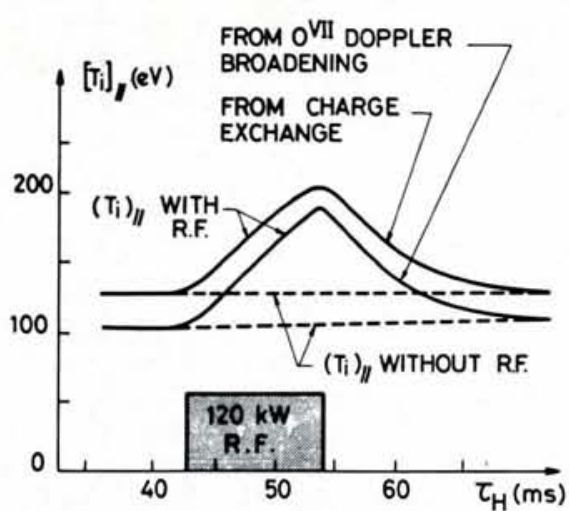

Fig. 4. Two determinations of $\left(T_{i}\right)_{/ /}$by charge exchange and Doppler broadening on the Tokamak WEGA.

(at the loffe Institute) and WEGA (at the Grenoble Laboratory), but the most conclusive results are those obtained very recently (September 1977) on the Tokamak WEGA. In this machine, by injecting an RF power larger than the ohmic heating, it has been shown that the ion temperature was doubled. The maximum RF power which was launched in WEGA was $180 \mathrm{~kW}$ without any perturbation of the M.H.D. equilibria and deleterious effects. The measurement made by charge exchange, Fig. 4, and Doppler broadening of OVII, CV, and CIV lines along the direction of the toroidal magnetic field demonstrate without doubt that the ions of the plasma bulk, near to the discharge axis are heated. The location of these lines in the plasma are deduced from the electron temperature measurement by Thomson scattering, Fig. 5. Their evolution proves that the RF energy is first deposited in the bulk, which is later cooled by the impurities coming from the walls. The perpendicular ion energy distribution has again an energetic tail, which does not produce disruptive discharge as in the case of I.C.R.H.

Further experiments at higher power are needed to extend the scaling law of this heating process.

\section{Future of RF heating}

On the basis of the present experimental situation, L.H.R.H., T.T.M.P. and finally I.C.R.H. are three leading candidates for providing the additional heating needed for a large thermonuclear device. Yet, due partly to the simpler physical mechanisms involved, successful preliminary tests and historical reasons, world effort in additional heating is concentrating on neutral beam injection. This solution requires however, an important source development and two questions remain still open: the efficiency and the tolerance of the neutral beam source to an intense neutron flux coming from the reactor.

The situation is more favourable in the case of an RF heating scheme. Indeed the development required for

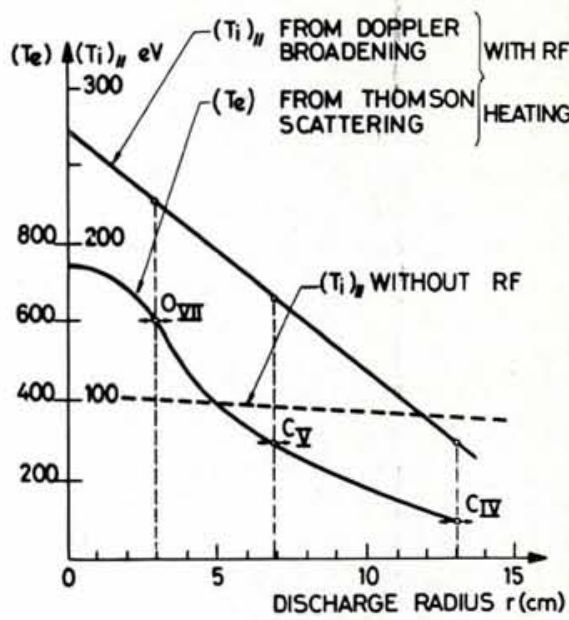

Fig. 5. Location of OVII, CV, CIV lines as a function of the electron temperature profile. $T_{e}(r)-\left(T_{i}\right)_{\|}$is maximum at the centre of the discharge.

a 50-100 MW emitter at low (f $<60$ $\mathrm{MHz}$ ) and medium ( $\mathrm{f}<3 \mathrm{GHz}$ ) frequencies is very moderate compared to the work required for neutral beam injection. Efficiency is higher and in contrast to a neutral beam source, an RF emitter can be put very far from the neutron flux, and the wave transmitted and launched with an RF line, coupled to the plasma preferably by external coils or by wave guides.

It is hoped that as further progress is made on RF heating, it will receive a bigger share of attention in future programmes.

\section{Non-Linear Optical Resonances}

\section{and their}

\section{Applications}

The production of narrow and frequency-stable resonances in absorption or emission spectra of substances over various ranges of electromagnetic radiation has always been an important problem in physics. Every discovery in this direction increases the accuracy of physical experiments and finds wide use in various fields of science and technology. Let us recall the two classical examples.

In the 1940 s and 1950 s, a technique was evolved of producing narrow resonances in the microwave frequency range using atomic and molecular beams. For example, Ramsey, using two separated electro-

\section{V.S. Letokhov, Moscow}

(Inst. of Spectroscopy of USSR Acad. of Sci.) magnetic fields interacting with a beam of $\mathrm{Cs}$ atoms at the transition between the hfs levels of the ground state, was able to obtain resonances with the width $\Delta v / v_{0}=5.10^{-9}$, in other words, with a "quality" (resolution) of resonance $R=v_{0} / \Delta v=2.10^{8}$. Narrow microwave resonance has formed the basis for quantum frequency standards and the universally adopted atomic time scale (atomic clocks).

Extremely narrow resonances in a higher-frequency region of the spectrum were detected at nuclear transitions by Mössbauer. For instance, at a $\gamma$-transition with the energy of 93

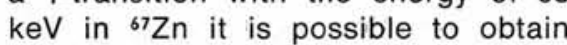
a resonance with the resolution of
2.1015. Narrow resonance of nuclear transitions without recoil in a crystal lattice now ensure the highest resolution in physical experiments, of the order of $10^{15}$.

In the intermediate (optical) spectral region, the relative resonance width until recently was no better than $10^{-6}$ because of Doppler broadening of spectral lines. The discovery of the subtle effects of resonant nonlinear interaction between the coherent light field and atomic or molecular gases described in this article, has allowed the relative widths of optical resonances to be narrowed by factors of $10^{-4}$ to $10^{-6}$. This has given impetus to the development of 